\title{
Ensemblization of density-functional theory: Insight from the fluctuation-dissipation theorem
}

\author{
Tim Gould \\ Qld Micro- and Nanotechnology Centre, Griffith University, Nathan, Qld 4111, Australia* \\ Gianluca Stefanucci \\ Dipartimento di Fisica, Università di Roma Tor Vergata, \\ Via della Ricerca Scientifica 1, 00133 Rome, Italy and \\ INFN, Sezione di Roma Tor Vergata, Via della Ricerca Scientifica 1, 00133 Rome, Italy \\ Stefano Pittalis \\ CNR - Istituto Nanoscienze, 41125 Modena, Italy
}

\begin{abstract}
Density functional theory can be generalized to mixtures of ground and excited states, for the purpose of determining energies of excitations using low-cost density functional approximations. Adapting approximations originally developed for ground states to work in the new setting would fast-forward progress enormously. But, previous attempts have stumbled on daunting fundamental issues. Here we show that these issues can be prevented from the outset, by using a fluctuation dissipation theorem (FDT) to dictate key functionals. We thereby show that existing exchange energy approximations are readily adapted to excited states, when combined with a rigorous exact Hartree term that is different in form from its ground state counterpart, and counterparts based on ensemble ansatzë. Applying the FDT to correlation energies also provides insights into ground state-like and ensemble-only correlations. We thus provide a comprehensive and versatile framework for ensemble density functional approximations.
\end{abstract}

Introduction: Averages and fluctuations are essential concepts to make sense of data of any sort. In physics, these quantities are also used to explore the formal relationships of theories and approximations. Via Feynman's path integrals [1], for example, classical physics itself can be seen to emerge in terms of an averaged path that, in the limit of $\hbar \rightarrow 0$, dominates over otherwise irreducible quantum fluctuations. In condensed-matter physics, to mention another important example, meanfield approximations are used to formalize the concept of the order parameters and their estimations; [2] consideration of fluctuations are then necessary to fully characterize second-order phase transitions.

Density functional theory (DFT) $[3,4]$ can also be conceptualized in terms of averages and fluctuations. In one and the same step [4] it overcomes the semiclassical Thomas-Fermi approximation [5] and the mean-field approximation by mapping the original many-body problem onto a one-electron problem capturing key fluctuations - the exchange and correlation (xc) terms in DFT parlance. Simple and effective xc approximations for these fluctuations have enabled DFT to become the workhorse of electronic structure calculations. [6]

In this Letter, we turn to a generalization of DFT through which excitation energies (not just the ground state energy) of a many-electron system can be computed; and invoke an extension of the (so-called) fluctuation dissipation theorem (FDT) to effectively deal with fluctuations relative to excited states. We shall refer to this formulation of DFT $[7,8]$ as "ensemble DFT" (EDFT) but, strictly, we deal with EDFT for excited states (rather than other formulations such as the one that accounts for states with different particle numbers [9]). EDFT is a primary competitor of linearresponse time-dependent DFT for the evaluation of excitation energies. Previous attempts at deriving improved approximations have, however, stumbled on a series of difficulties. Progress has recently attained a faster pace due to new fundamental and practical results [10-27].

Current wisdom stresses that ensembles are best dealt with by treating Hartree-Fock (Hartree-exchange, Hx, in DFT) energies as a "conjoint" unit. [10, 18] Indeed, two of the authors have previously strongly espoused this approach. [21] But, much of the progress in devising density functional approximations (DFA) for ground states has been enabled by treating Hartree $(\mathrm{H})$ and exchange $(\mathrm{x})$ components on different footings: with the former almost always employed in its exact form and the latter approximated in full, $E_{\mathrm{Hxc}} \approx E_{\mathrm{H}}+E_{\mathrm{xc}}^{\mathrm{DFA}}$, (standard approximations) or partially, $E_{\mathrm{Hxc}} \approx \alpha E_{\mathrm{Hx}}+(1-\alpha)\left(E_{\mathrm{H}}+\right.$ $\left.E_{\mathrm{x}}^{\mathrm{DFA}}\right)+E_{\mathrm{c}}^{\mathrm{DFA}}$ (hybrid approximations). DFAs of this form have been refined over decades to balance accuracy and practicality. It is imperative that we can transfer such experience to excited states. Attempts so far have been positive, but of somewhat narrow scope. Has the time come to surrender?

Quite on the contrary, here we show that the 'Hx' energy in EDFT can be split into disjoint Hartree-like and exchange-like contributions, which avoid the disastrous issues encountered in previous proposals. We derive this decomposition with the help of a generalization of the FDT to passive states - ensemble states whose weights 
are non-increasing with increasing energy and are thereby amenable to EDFT treatment. [28] The same FDT also identifies key components of the corresponding correlation energy functional. Hence we draw a complete, user friendly, theoretical roadmap for developing novel approximations for excited states - which builds from fundamental principles without sacrificing previous work.

We begin with a brief introduction to EDFT for excited states; and illustrate the problem that is solved in this work. Then, we work out the details of the main results and see them in action on prototypical cases. We first work on the Hartree and exchange energy functionals, then complete the development by deriving corresponding results for correlations, before concluding.

Crash-course in EDFT: Consider the Hamiltonian $\hat{H}=\hat{T}+\hat{W}+\hat{V}_{\text {ext }}$ where $\hat{T}$ is the kinetic energy of the particles (electrons, in our case), $\hat{W}$ is the two-body interaction (typically the Coulomb interaction), and $\hat{V}_{\text {ext }}$ is an external potential (typically the scalar electric potential exerted by atomic nuclei on electrons).

We are often interested in a finite set of states with different energies (or symmetries when degenerate) but the same number of electrons - typically a handful of low-lying solutions of $\hat{H}|\kappa\rangle=E_{\kappa}|\kappa\rangle$. These form the ensemble described by the operator $\hat{\Gamma}^{\boldsymbol{w}}=\sum_{\kappa} w_{\kappa}|\kappa\rangle\langle\kappa|$ where a set of prescribed mixing weights $w_{\kappa} \geq 0$ (collectively, $\boldsymbol{w})$ are taken as non-increasing (i.e., $w_{\kappa} \leq w_{\kappa^{\prime}}$ for $E_{\kappa} \geq E_{\kappa^{\prime}}$ ) and normalised $\sum_{\kappa} w_{\kappa}=1$. Such ensembles belong to the class of passive states [29]. Issues due to spurious breaking of symmetries are avoided by assigning equal weights to multiplets to obtain a totally symmetric passive state. [30] The average of an operator, $\hat{O}$, can be written as, $\operatorname{Tr}\left[\hat{\Gamma}^{\boldsymbol{w}} \hat{O}\right]$.

$\hat{\Gamma}^{\boldsymbol{w}}$ fulfills an extended variational principle [28] according to which

$$
\operatorname{Tr}\left[\hat{\Gamma}_{\text {trial }}^{\boldsymbol{w}} \hat{H}\right] \geq \mathcal{E}=\operatorname{Tr}\left[\hat{\Gamma}^{\boldsymbol{w}} \hat{H}\right] .
$$

Thus, $\mathcal{E}=\inf _{\hat{\Gamma}_{\text {trial }}^{w}} \operatorname{Tr}\left[\hat{\Gamma}_{\text {trial }}^{\boldsymbol{w}} \hat{H}\right]$ where the argument for the infimum (usually a minimum), $\hat{\Gamma}_{\text {trial }}^{\boldsymbol{w}}=\sum_{\kappa} w_{\kappa}\left|\kappa_{t}\right\rangle\left\langle\kappa_{t}\right|$, involves prescribed weights, $\boldsymbol{w}$, and mutually orthonormal trial wavefunctions $\left|\kappa_{t}\right\rangle$.

In EDFT we compute the average energy, $\mathcal{E}$, by minimizing the following functional of the ensemble-particle density, $n,[7,8]$

$$
\mathcal{E}^{\boldsymbol{w}}[n]=\mathcal{T}_{s}^{\boldsymbol{w}}[n]+\mathcal{E}_{\mathrm{Hxc}}^{\boldsymbol{w}}[n]+\int n(\boldsymbol{r}) v_{\mathrm{ext}}(\boldsymbol{r}) d \boldsymbol{r} .
$$

By analogy with standard DFT, $\mathcal{T}_{s}^{\boldsymbol{w}}[n]=$ $\min _{\hat{\Gamma}_{\text {trial }}^{w} \rightarrow n} \operatorname{Tr}\left[\hat{\Gamma}_{\text {trial }}^{\boldsymbol{w}} \hat{T}\right]$, is the kinetic energy of the Kohn-Sham (KS) system reproducing the particle density of the ensemble; the minimum is attained at $\hat{\Gamma}_{s} \equiv \sum_{\kappa} w_{\kappa}\left|\kappa_{s}\right\rangle\left\langle\kappa_{s}\right| .[31] \mathcal{E}_{\mathrm{Hxc}}^{\boldsymbol{w}}[n]$ subsumes the Hartree, exchange, and correlation energies for the ensemble. Note that in writing the energy functionals we use capital calligraphic letters to refer to energies of mixed states and capital Roman letters to refer to energies of pure state or their components, respectively. The superscript $\boldsymbol{w}$ indicates quantities that explicitly depend on the weights, meaning eq. (2) describes a different functional for every choice of $\boldsymbol{w}$. Varying $\boldsymbol{w}$ lets us, e.g., obtain excitation energies. The superscript shall henceforth be dropped for brevity.

Thus one can define a KS Hamiltonian $\hat{H}_{s}=\hat{T}+\hat{V}_{s}[n]$ which yields the same ensemble density, $n=\operatorname{Tr}\left[\hat{\Gamma}_{s} \hat{n}\right]=$ $\operatorname{Tr}[\hat{\Gamma} \hat{n}]$, as the interacting system when the KS ensemble operator $\hat{\Gamma}_{s} \equiv \sum_{\kappa} w_{\kappa}\left|\kappa_{s}\right\rangle\left\langle\kappa_{s}\right|$ minimizes $\operatorname{Tr}\left[\hat{\Gamma}_{s} \hat{H}_{s}\right]$. Following standard derivations one can show that the effective KS potential is given by $v_{s} \equiv v_{\text {ext }}+\frac{\delta \mathcal{E}_{\mathrm{Hxc}}}{\delta n}$. We emphasize that ambiguities in constructing $\hat{\Gamma}_{s}$ due to extra (noninteracting) degeneracies are removed by choosing the $\kappa_{s}$ 's to belong to the same irreducible representation as the $\kappa$ 's; hence, the $\kappa_{s}$ 's may be configuration-state functions (CSFs) (i.e., finite linear combinations of Slater determinants). This allows us to preserve all the relevant symmetries, consistently. [32]

In particular, because we work at the level of totally symmetric ensembles, ensembles KS densities and corresponding KS kinetic energies can still be computed by referring to a "minimal" set of single Slater determinants, $\left\{\Phi_{\kappa}\right\}$, from which all the relevant $\left|\kappa_{s}\right\rangle$ states can be constructed. Therefore, $n \equiv \sum_{i} f_{i}\left|\phi_{i}\right|^{2}$, and, $\mathcal{T}_{s}=$ $-\frac{1}{2} \sum_{i} f_{i} \int d \boldsymbol{r} \phi_{i}^{*} \nabla^{2} \phi_{i}$, involve one-body orbitals obeying the ensemble KS equation, $\left\{-\frac{1}{2} \nabla^{2}+v_{s}[n](\boldsymbol{r})\right\} \phi_{i}(\boldsymbol{r})=$ $\epsilon_{i} \phi_{i}(\boldsymbol{r})$. Differences from ground state KS theory appear in the fractional occupation factors, $f_{i}=\sum_{\kappa} w_{\kappa} \theta_{i, \kappa}$, which are ensemble averages of total ( $\uparrow, \downarrow$ or $\uparrow \downarrow)$ occupation factors, $\theta_{i, \kappa}$, for orbital $i$ in state $\Phi_{\kappa}$; and also in the form of the Hxc energy functional, $\mathcal{E}_{\mathrm{Hxc}}$ (see later).

A long standing struggle: Recall, in DFT we define the Hartree energy as,

$$
E_{\mathrm{H}}[n] \equiv \frac{1}{2} \int d \boldsymbol{r} d \boldsymbol{r}^{\prime} \frac{n(\boldsymbol{r}) n\left(\boldsymbol{r}^{\prime}\right)}{\left|\boldsymbol{r}-\boldsymbol{r}^{\prime}\right|}
$$

and, correspondingly, the exchange energy as,

$$
E_{\mathrm{x}}[n] \equiv-\frac{1}{2} \int d \boldsymbol{r} d \boldsymbol{r}^{\prime} \frac{\left|\rho_{s}\left(\boldsymbol{r}, \boldsymbol{r}^{\prime}\right)\right|^{2}}{\left|\boldsymbol{r}-\boldsymbol{r}^{\prime}\right|} \equiv E_{\mathrm{x}}\left[\rho_{s}\right] .
$$

Here, $\rho_{s}\left(\boldsymbol{r}, \boldsymbol{r}^{\prime}\right)=\sum_{i} f_{i} \phi_{i}^{*}(\boldsymbol{r}) \phi_{i}\left(\boldsymbol{r}^{\prime}\right)$ is the KS one-body reduced density matrix (1-RDM) obeying $\rho_{s}(\boldsymbol{r}, \boldsymbol{r})=n(\boldsymbol{r})$. Note that $\rho_{s}\left(\boldsymbol{r}, \boldsymbol{r}^{\prime}\right)$ is a functional of the particle density, $\rho_{s}\left(\boldsymbol{r}, \boldsymbol{r}^{\prime}\right)=\rho_{s}[n]\left(\boldsymbol{r}, \boldsymbol{r}^{\prime}\right)$, because the KS orbitals have an implicit dependency on $n$.

A natural ansatz (Ans1) to extend the above equations to EDFT is to replace the density and 1-RDM by their ensemble versions. Then, $\mathcal{E}_{\mathrm{H}}^{\text {Ans1 }}[n]:=E_{\mathrm{H}}\left[\sum w_{\kappa} n_{s, \kappa}(\boldsymbol{r})\right]=$ $E_{\mathrm{H}}\left[\sum f_{i}\left|\phi_{i}(\boldsymbol{r})\right|^{2}\right]$ and $\mathcal{E}_{\mathrm{x}}^{\mathrm{Ans} 1}[n]:=E_{\mathrm{x}}\left[\sum w_{\kappa} \rho_{s, \kappa}\left(\boldsymbol{r}, \boldsymbol{r}^{\prime}\right)\right]=$ $E_{\mathrm{x}}\left[\sum f_{i} \phi_{i}^{*}(\boldsymbol{r}) \phi_{i}\left(\boldsymbol{r}^{\prime}\right)\right]$, where $\rho_{s, \kappa}=\left\langle\kappa_{s}|\hat{\rho}| \kappa_{s}\right\rangle$ (similarly for $\left.n_{s, \kappa}\right)$. Here, both $n_{s, \kappa}$ and $\rho_{s, \kappa}$ are functionals of the overall density $n$. Ans1 is appealing because it allows standard DFA to be readily reused in EDFT $[7,8]$. 
But, in practice, Ans1 has disastrous consequences. "Ghost interaction errors" (GIE) badly affect both Hartree and exchange, but do not cancel each other. GIE can be understood as a generalization of the one-particle self-interaction error, in which electrons spuriously interact with 'ghost' counterparts in a different replica in the ensemble, leading to errors. GIE corrections have traditionally been worked out ad hoc. [13, 33, 34] Recent work $[17,26]$ has made interesting progress in using Ans1 together with weight-dependent xc-approximations.

GIE can sometimes be avoided by considering a different and, arguably, better ansatz (Ans2),

$$
\begin{aligned}
& \mathcal{E}_{\mathrm{H}}^{\mathrm{Ans} 2}[n]:=\sum_{\kappa} w_{\kappa} E_{\mathrm{H}}\left[n_{s, \kappa}\right], \\
& \mathcal{E}_{\mathrm{x}}^{\text {Ans2 }}[n]:=\sum_{\kappa} w_{\kappa} E_{\mathrm{x}}\left[\rho_{s, \kappa}\right] .
\end{aligned}
$$

Ans2 is appealling, as it suggests that ground state functionals may be upgraded to ensembles via weighted averages of their "clones". Ans2, however, falls short in certain cases, such as difficult spin-multiplets (see below).

All issues with the previous definitions for $\mathcal{E}_{\mathrm{H}}[n]$ and $\mathcal{E}_{\mathrm{x}}[n]$ are resolved by working at the level of a conjoint $\mathcal{E}_{\mathrm{Hx}}[n]$ which is problem-free by construction: [21]

$$
\mathcal{E}_{\mathrm{Hx}}[n] \equiv \lim _{\lambda \rightarrow 0^{+}} \frac{\mathcal{F}^{\lambda}[n]-\mathcal{T}_{s}[n]}{\lambda} \equiv \operatorname{Tr}\left[\hat{\Gamma}^{0^{+}} \hat{W}\right] .
$$

Eq. (7) is successful (and maximally GIE free) because it is written in terms of the well-defined universal functional $\mathcal{F}^{\lambda}[n]=\min _{\hat{\Gamma} \rightarrow n} \operatorname{Tr}[\hat{\Gamma}(\hat{T}+\lambda \hat{W})] \equiv \operatorname{Tr}\left[\hat{\Gamma}^{\lambda}(\hat{T}+\lambda \hat{W})\right]$, where $\hat{\Gamma}^{\lambda}=\sum w_{\kappa}\left|\kappa^{\lambda}\right\rangle\left\langle\kappa^{\lambda}\right|$ involves eigen-solutions, $\left|\kappa^{\lambda}\right\rangle$, of $\left\{\hat{T}+\lambda \hat{W}+\hat{V}^{\lambda}\right\}\left|\kappa^{\lambda}\right\rangle=E_{\kappa}^{\lambda}\left|\kappa^{\lambda}\right\rangle$; with $v^{\lambda}[n]$ chosen to ensure $n=\operatorname{Tr}\left[\hat{\Gamma}^{\lambda} \hat{n}\right]$ regardless of $\lambda$. When totally symmetric ensembles are employed, $\hat{\Gamma}^{\lambda}[n]$, is also a unique functional of $n$ and $\boldsymbol{w}$ [24] so we restrict to this useful case. We also assume that the ordering of states is preserved (or preservable) along the adiabatic path $0<\lambda \leq 1$. [35] Note, we can now rigorously define $\hat{\Gamma}_{s} \equiv \lim _{\lambda \rightarrow 0^{+}} \hat{\Gamma}^{\lambda} \equiv \hat{\Gamma}^{0^{+}}, \mathcal{T}_{s} \equiv \mathcal{F}^{0}$ and $\mathcal{E}_{\mathrm{Hxc}} \equiv \mathcal{F}^{1}-\mathcal{T}_{s}$.

The conjoint treatment leads to well-defined "ensemblized" versions of Hartree-exchange [21] and correlations [23], albeit with some additional complications compared to the ground state. But, keeping ' $H$ ' and ' $x$ ' joint together loses the long experience gained in DFAs which treat them separately. This is the most urgent drawback of eq. (7) as it hampers access to prior work.

Its impact can be easily seen by considering the following ensemble generalization of conventional DFAs:

$$
\mathcal{E}_{\mathrm{xc}}^{\mathrm{DFA}}[n]:=\sum_{\kappa} w_{\kappa} E_{\mathrm{xc}}^{\mathrm{DFA}}\left[n_{s, \kappa}\right] .
$$

Eq. (8) involves an ensemble average of a standard DFA (e.g. PBE) for xc, along the lines of Ans2. It can thus draw from decades of work on DFA development. But, it does not correct for all ghost interactions. Evaluating the ensemble energy, $\mathcal{E}=\mathcal{T}_{s}+\mathcal{E}_{\mathrm{H}}+\mathcal{E}_{\mathrm{xc}}^{\mathrm{DFA}}+\int d \boldsymbol{r} n v_{\text {ext }}+\mathcal{E}_{\mathrm{c}}^{\mathrm{DD}}$ (the final term is discussed below) therefore requires choosing a GIE-free Hartree term to pair with eq. (8). Efforts to develop new approximations can thus be fastforwarded, by ensuring that $\mathcal{E}_{\mathrm{H}}$ captures the energy components that would otherwise be missed. Below, we will show that the ideal $\mathcal{E}_{\mathrm{H}}$ must go beyond $\mathcal{E}_{\mathrm{H}}^{\text {Ans2 }}$. Furthermore, our derivation also sheds light on, $\mathcal{E}_{\mathrm{c}}^{\mathrm{DD}}$ : the recently discovered "density-driven" correlation energy, [23] discussed in recent work. [23, 25]

Resolution: First, recall that the average of the interaction can be expressed as follows: $\operatorname{Tr}\left[\hat{\Gamma}^{\lambda} \hat{W}\right] \equiv \int \frac{d \boldsymbol{r} d \boldsymbol{r}^{\prime}}{2\left|\boldsymbol{r}-\boldsymbol{r}^{\prime}\right|}$ $n_{2}^{\lambda}\left(\boldsymbol{r}, \boldsymbol{r}^{\prime}\right)$ where, $n_{2}^{\lambda}\left(\boldsymbol{r}, \boldsymbol{r}^{\prime}\right)=\operatorname{Tr}\left[\hat{\Gamma}^{\lambda} \hat{n}(\boldsymbol{r}) \hat{n}\left(\boldsymbol{r}^{\prime}\right)\right]-n(\boldsymbol{r}) \delta(\boldsymbol{r}-$ $\left.\boldsymbol{r}^{\prime}\right)$. The crucial second step is to use the FDT for passive states - stated and proved in the Supplementary Material [36] - to write,

$$
\operatorname{Tr}\left[\hat{\Gamma}^{\lambda} \hat{n}(\boldsymbol{r}) \hat{n}\left(\boldsymbol{r}^{\prime}\right)\right]=P^{\lambda}\left(\boldsymbol{r}, \boldsymbol{r}^{\prime}\right)-\int_{0^{-}}^{\infty} \frac{d \omega}{\pi} \Im \chi^{\lambda}\left(\omega, \boldsymbol{r}, \boldsymbol{r}^{\prime}\right),
$$

in terms of the ensemble (retarded density-density) response, $\chi^{\lambda}$, of the considered passive state, $\hat{\Gamma}^{\lambda}$; and a relevant pair-density,

$$
P^{\lambda}\left(\boldsymbol{r}, \boldsymbol{r}^{\prime}\right)=\sum_{\kappa \kappa^{\prime}} \min \left[w_{\kappa}, w_{\kappa^{\prime}}\right] n_{\kappa \kappa^{\prime}}^{\lambda}(\boldsymbol{r}) n_{\kappa^{\prime} \kappa}^{\lambda}\left(\boldsymbol{r}^{\prime}\right),
$$

where $n_{\kappa \kappa^{\prime}}^{\lambda} \equiv\left\langle\kappa^{\lambda}|\hat{n}| \kappa^{\prime \lambda}\right\rangle$. Note, $P^{\lambda}\left(\boldsymbol{r}, \boldsymbol{r}^{\prime}\right) \equiv \sum_{\kappa} w_{\kappa}$ $n_{\kappa \kappa}^{\lambda}(\boldsymbol{r}) n_{\kappa \kappa}^{\lambda}\left(\boldsymbol{r}^{\prime}\right)+\sum_{\kappa^{\prime}<\kappa} w_{\kappa}\left[n_{\kappa \kappa^{\prime}}^{\lambda}(\boldsymbol{r}) n_{\kappa^{\prime} \kappa}^{\lambda}\left(\boldsymbol{r}^{\prime}\right)+\boldsymbol{r} \leftrightarrow \boldsymbol{r}^{\prime}\right]$ entails two terms: the first term resembles a typical meanfield-like contribution and involves products of pure state densities $\left(n_{\kappa \kappa}^{\lambda}\right)$; the second involves off-diagonal matrix elements $\left(n_{\kappa \neq \kappa^{\prime}}^{\lambda}\right)$ which represent extra ensemble-specific fluctuations that are absent in pure ground states.

Next, by invoking the analogous FDT relationships from ground state DFT functionals we can establish the EDFT counterparts that account for degeneracies and excited states. Any non-degenerate ground state obeys $E_{\mathrm{x}}=-\int \frac{d \boldsymbol{r} d \boldsymbol{r}^{\prime}}{2\left|\boldsymbol{r}-\boldsymbol{r}^{\prime}\right|}\left[n(\boldsymbol{r}) \delta\left(\boldsymbol{r}-\boldsymbol{r}^{\prime}\right)+\int_{0}^{\infty} \frac{d \omega}{\pi} \Im \chi_{s}\left(\omega, \boldsymbol{r}, \boldsymbol{r}^{\prime}\right)\right]$, where $\chi_{s}$ is the KS response of the ground state. This relationship is useful for constructing and understanding DFAs. It is therefore natural to similarly define,

$$
\begin{aligned}
\mathcal{E}_{\mathrm{x}}^{\mathrm{FDT}}[n] \equiv-\int \frac{d \boldsymbol{r} d \boldsymbol{r}^{\prime}}{2\left|\boldsymbol{r}-\boldsymbol{r}^{\prime}\right|}\left\{n(\boldsymbol{r}) \delta\left(\boldsymbol{r}-\boldsymbol{r}^{\prime}\right)\right. & \\
& \left.+\int_{0^{-}}^{\infty} \frac{d \omega}{\pi} \Im \chi_{s}[n]\left(\boldsymbol{r}, \boldsymbol{r}^{\prime} ; \omega\right)\right\},
\end{aligned}
$$

where $\chi_{s} \equiv \chi^{\lambda=0^{+}}$is the ensemble KS response function for the considered passive state, $\hat{\Gamma}^{0^{+}}$. Finally, using the FDT [eq. (9)] with eq. (7) dictates the form of the corresponding EDFT Hartree functional: $\mathcal{E}_{\mathrm{H}}^{\mathrm{FDT}}=\mathcal{E}_{\mathrm{Hx}}-\mathcal{E}_{\mathrm{x}}^{\mathrm{FDT}}$, or,

$$
\mathcal{E}_{\mathrm{H}}^{\mathrm{FDT}}[n]=\int \frac{d \boldsymbol{r} d \boldsymbol{r}^{\prime}}{2\left|\boldsymbol{r}-\boldsymbol{r}^{\prime}\right|} P_{s}[n]\left(\boldsymbol{r}, \boldsymbol{r}^{\prime}\right)
$$


$P_{s} \equiv P^{\lambda=0^{+}}$is defined via eq. (10) with $n_{s, \kappa \kappa^{\prime}} \equiv n_{\kappa \kappa^{\prime}}^{\lambda=0^{+}}$. Thus, only non-interacting KS states are involved. [21, 24] $P^{\lambda}[n]$ and $\chi^{\lambda}[n]$ are uniquely defined by $v^{\lambda}[n]$ and $\boldsymbol{w}$, meaning eqs (9)-(12) are functionals of the density.

Eq. (12) reduces to the usual semi-classical mean-field approximation for a non-degenerate ground state (gs), with $P_{s}\left[n_{\mathrm{gs}}\right]\left(\boldsymbol{r}, \boldsymbol{r}^{\prime}\right)=n_{\mathrm{gs}}(\boldsymbol{r}) n_{\mathrm{gs}}\left(\boldsymbol{r}^{\prime}\right)$, since $w_{\mathrm{gs}}=1$ and $w_{\kappa}=0$ otherwise. But in general, eq. (12) involves products between off-diagonal matrix elements of the density, from eq. (10). Disregarding these off-diagonal terms, i.e., replacing $P_{s}$ by $\sum_{\kappa} w_{\kappa} n_{s, \kappa \kappa}(\boldsymbol{r}) n_{s, \kappa \kappa}\left(\boldsymbol{r}^{\prime}\right)$, reduces $\mathcal{E}_{\mathrm{H}}^{\mathrm{FDT}}$ to $\mathcal{E}_{\mathrm{H}}^{\text {Ans2 }}$ and involves direct integral terms only. The offdiagonal terms lead to additional exchange integrals in $\mathcal{E}_{\mathrm{H}}^{\mathrm{FDT}}$ which, because $\mathcal{E}_{\mathrm{H}}^{\mathrm{FDT}}+\mathcal{E}_{\mathrm{x}}^{\mathrm{FDT}}=\mathcal{E}_{\mathrm{Hx}}$, must provide contributions that are missed by Ans2. Applying SlaterCondon rules to eq. (7) must give equivalent terms - the FDT assigns these terms unambiguously to $\mathrm{H}$ or $\mathrm{x}$.

Last but not least, an additional nice property of $\mathcal{E}_{\mathrm{H}}^{\mathrm{FDT}}$ and $\mathcal{E}_{\mathrm{x}}^{\mathrm{FDT}}$ is that their integrands are invariant to rotations around axes of symmetry, in contrast to Ans2 which has an unphysical dependence on the choice of axes. Details are in the Supplementary Material. [36]

Illustrative examples: To keep the discussion of key results simple, let us work with a two electron system. In KS theory, the ground state has a doubly occupied orbital, $\phi_{0}$, whereas the low-lying singlet/triplet excitations involve $\phi_{0}$ and $\phi_{1}$ together.

First, consider $\hat{\Gamma}_{s}^{\mathrm{ST}}=w_{\mathrm{S}}\left|\mathrm{S}_{0}\right\rangle\left\langle\mathrm{S}_{0}\right|+w_{\mathrm{T}} \hat{\Gamma}_{\mathrm{T}}$ consisting of the KS singlet ground state $\left(\mathrm{S}_{0}\right)$ and the lowest-lying KS triplet $\hat{\Gamma}_{s}^{\mathrm{T}}=\frac{1}{3} \sum_{\mathrm{S}_{\mathrm{z}}=1,0,-1}\left|\mathrm{~T}_{\mathrm{S}_{\mathrm{z}}}\right\rangle\left\langle\mathrm{T}_{\mathrm{S}_{\mathrm{z}}}\right|$ mixed with weights $w_{\mathrm{T}} \leq \frac{1}{2}$ and $w_{\mathrm{S}}=1-w_{\mathrm{T}}$. Because $\hat{n}$ is spin independent, $\left\langle\mathrm{T}_{\mathrm{S}_{\mathrm{z}}}|\hat{n}| \mathrm{S}_{0}\right\rangle=0$. Hence, eq. (12) yields $\mathcal{E}_{\mathrm{H}, \mathrm{ST}}^{\mathrm{FDT}}=\mathcal{E}_{\mathrm{H}, \mathrm{ST}}^{\text {Ans2 }}$.

Then, consider $\hat{\Gamma}_{s}^{\mathrm{SS}}=w_{\mathrm{S}_{0}}\left|\mathrm{~S}_{0}\right\rangle\left\langle\mathrm{S}_{0}\left|+w_{\mathrm{S}_{1}}\right| \mathrm{S}_{1}\right\rangle\left\langle\mathrm{S}_{1}\right|$, which mixes the ground $\left(\mathrm{S}_{0}\right)$ state, as above, with the first KS excited singlet state $\left(\mathrm{S}_{1}\right)$, with weights $w_{\mathrm{S}_{1}} \leq \frac{1}{2}$ and $w_{\mathrm{S}_{0}}=1-w_{\mathrm{S}_{1}}$. This ensemble enables direct evaluation of excitations of the same symmetry. Eq. (12) yields,

$$
\mathcal{E}_{\mathrm{H}, \mathrm{SS}}=\mathcal{E}_{\mathrm{H}, \mathrm{SS}}^{\mathrm{Ans} 2}+4 w_{S_{1}} E_{\mathrm{H}}\left[\phi_{0} \phi_{1}\right] ;
$$

i.e., eq. (13) differs, in its final term, from the weighted average of Ans2. Ans2 and FDT both yield orbital functionals and thus require ensemble generalized Kohn-Sham theories [37], optimized-effective potentials (OEP) [38-40] or approximations. [17, 24, 27, 40]

Finally, consider the SS and ST ensembles when the ground state has the same weight $1-w$. For simplicity, also allow the orbitals to be the same. It follows that $\mathcal{E}_{\mathrm{H}, \mathrm{SS}}^{\mathrm{FDT}}=\mathcal{E}_{\mathrm{H}, \mathrm{ST}}^{\mathrm{FDT}}+4 w E_{\mathrm{H}}\left[\phi_{0} \phi_{1}\right]$. The Hartree difference for the SS and ST ensembles is the (weighted) spin splitting energy, $4 E_{\mathrm{H}}\left[\phi_{0} \phi_{1}\right]$, obtained at the level of CSFs - which is therefore captured when eq. (12) is combined with eq. (8). This is not surprising given that direct and exchange integrals appear in $\mathcal{E}_{\mathrm{H}}^{\mathrm{FDT}}$. In contrast, $\mathcal{E}_{\mathrm{H}, \mathrm{SS}}^{\mathrm{Ans} 2}=\mathcal{E}_{\mathrm{H}, \mathrm{ST}}^{\mathrm{Ans}}$ does not contain this term.

Let us now test how the choice of Hartree functional

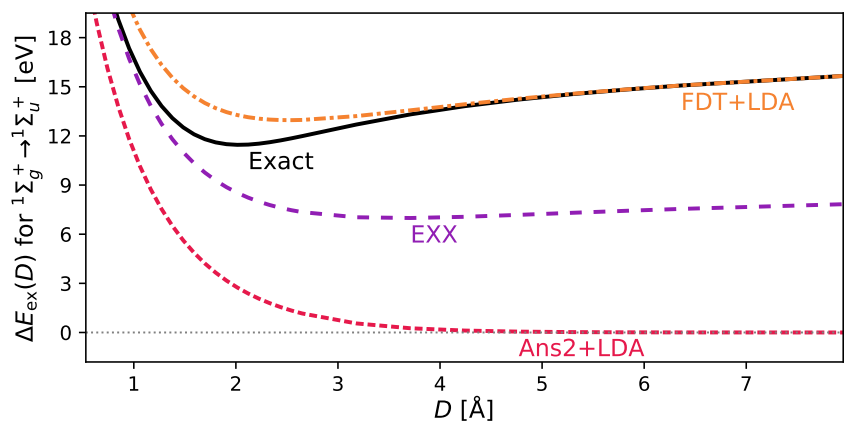

FIG. 1. SS excitation $\left({ }^{1} \Sigma_{g}^{+} \rightarrow{ }^{1} \Sigma_{u}^{+}\right)$energies, $\Delta E_{\text {ex }}(D)$ for $\mathrm{H}_{2}$, as a function of interatomic distance, $D$. Compares FDT+LDA (orange dash dot) and Ans2+LDA (red short dashes) against exact energies (black) and EXX (blue dashes).

affects energies. To this end, Figure 1 shows the SS excitation energy, $\Delta E_{\mathrm{ex}}$, of dissociating $\mathrm{H}_{2}$ in a minimal basis set, using the local density approximation (LDA, $\left.E_{\mathrm{xc}}^{\mathrm{LDA}}\right)$ in eq. (8), paired with $\mathcal{E}_{\mathrm{H}}^{\mathrm{FDT}}(\mathrm{FDT}+\mathrm{LDA})$ or $\mathcal{E}_{\mathrm{H}}^{\text {Ans2 }}$ (Ans2+LDA). This simple, non-trivial example illustrates differences between the two Hartree functionals but avoids complexities like weight-dependence and OEPs. Details are in the Supplementary Material. [36]

FDT+LDA has a clear (albeit imperfect) minima and agrees perfectly with exact calculations for large $D$ $\left(\Delta E_{\text {ex }} \rightarrow 18 \mathrm{eV}\right)$. In contrast, Ans2+LDA predicts no minima and no gap for $D \rightarrow \infty$. Ans1, not shown, is nearly identical to Ans2. Exact exchange (EXX, $\mathcal{E}_{\mathrm{Hxc}} \approx \mathcal{E}_{\mathrm{Hx}}$ ) underestimates the asymptotic gap by a factor of two $\left(\Delta E_{\text {ex }} \rightarrow 9 \mathrm{eV}\right)$ and has an extremely shallow minima. For this example, it is clear that only FDT+LDA produces qualitatively correct physics.

General purpose ensemble DFAs must go beyond the LDA in eq. (8). They must also deal with density-driven correlations. [23, 25] The penultimate section thus applies the FDT to the correlation energy functional, $\mathcal{E}_{\mathrm{c}}$, to reveal its inner components, for the purpose of devising and improving ensemble DFAs.

Correlations: Let us adiabatically connect the noninteracting $\left(\lambda=0^{+}\right)$and fully interacting $(\lambda=1)$ systems. The Hellmann-Feynman theorem lets us write, $\mathcal{E}_{\mathrm{Hxc}} \equiv \mathcal{F}^{1}-\mathcal{F}^{0^{+}}=\int_{0^{+}}^{1} d \lambda \frac{d \mathcal{F}^{\lambda}}{d \lambda}=\int_{0^{+}}^{1} d \lambda \operatorname{Tr}\left[\hat{\Gamma}^{\lambda} \hat{W}\right] . \mathrm{We}$ can then subtract the corresponding Hx contribution (derived earlier) to obtain the correlation energy,

$$
\begin{gathered}
\mathcal{E}_{\mathrm{c}}[n] \equiv \int_{0^{+}}^{1} d \lambda \int \frac{d \boldsymbol{r} d \boldsymbol{r}^{\prime}}{2\left|\boldsymbol{r}-\boldsymbol{r}^{\prime}\right|}\left\{\Delta P^{\lambda}\left(\boldsymbol{r}, \boldsymbol{r}^{\prime}\right)\right. \\
\left.-\int_{0^{-}}^{\infty} \frac{d \omega}{\pi} \Im \Delta \chi^{\lambda}\left(\boldsymbol{r}, \boldsymbol{r}^{\prime} ; \omega\right)\right\}
\end{gathered}
$$

Therefore, we see that, $\mathcal{E}_{\mathrm{c}}$ entails two key contributions: a contribution, $\Delta P^{\lambda}[n]=P^{\lambda}[n]-P_{s}[n]$, that naturally pairs with $\mathcal{E}_{\mathrm{H}}^{\mathrm{FDT}}$; and a second contribution, $\Delta \chi^{\lambda}[n]=$ $\chi^{\lambda}[n]-\chi_{s}[n]$, that naturally pairs with $\mathcal{E}_{\mathrm{x}}^{\mathrm{FDT}}$. 
First of all, note that $\Delta P_{\mathrm{gs}}^{\lambda} \equiv 0$ in pure state DFT because the density, $n \equiv n_{\mathrm{gs}}$, is preserved along the adiabatic connection. In EDFT only the total ensemble density $n=\sum w_{\kappa} n_{\kappa \kappa}^{\lambda}$ is preserved, whereas the densities of individual terms, $n_{\kappa \kappa^{\prime}}^{\lambda}$, can (and do $[22,23]$ ) vary. Some of the present authors already stressed that this implies a new form a correlation in EDFT: density-driven (DD) correlations, the relevance of which has been thoroughly illustrated in previous works. [23, 25] The contribution from $\Delta P^{\lambda}$ thus provides an explicit expression for part of the DD correlations.

The contribution from $\Delta \chi^{\lambda}$ is expressed as a (correlation) density functional of the "adiabatic-connection fluctuations-dissipation" (ACFD) variety [41-44] - here extended to passive states. ACFD approximations are used directly and as an ingredient in "double hybrid" approximations. [45] The integral over frequency reduces sensitivity of ACFD approximations to issues like "memory effects" that manifest when using response functions to directly evaluate excitation energies. Whether these good features hold for ensembles should be explored in future work.

Conclusions: The "Hartree-exchange" energy functional for ensembles [eq. (7)] is a wilder beast than its ground state counterpart. Here, we have shown that it can be tamed by using the fluctuation-dissipation theorem to define an exchange functional [eq. (11)] that is amenable to conventional approximations; and thus dictate an unconventional Hartree functional [eq. (12)] whose extra terms account for spin-multiplets and other symmetry issues. The correlation energy has a similar division [eq. (14)], which provides insights into densitydriven correlations. [23, 25]

This work thus completes the 'ensemblization' of density functional theory that was begun in previous works; [21, 23] and provides a comprehensive ensemble treatment of all key functionals. It reveals how years of successful experience accumulated for ground states can, in principle, be re-used in a problem-free fashion for excitations. Future work should investigate how the framework provided here can be used to develop successful approximations, especially for double (and higher multiple) excitations that are difficult to capture in TDDFT. [46, 47] Its relationship to other EDFT formalisms [16, 25] should also be investigated.

Acknowledgments: TG acknowledges funding from ARC DP200100033. GS acknowledges funding from MIUR PRIN Grant No. 20173B72NB and from INFN17Nemesys project. SP acknowledges funding from MIUR PRIN Grant No. 2017RKWTMY.

* t.gould@griffith.edu.au

[1] R. P. Feynman and A. R. Hibbs, Quantum Mechanics and Path Integrals (Dover Publications, INC., Mineola, New York, 2010) emended Edition.

[2] P. Anderson, Basic notions of condensed matter physics. (Addison-Wesley, Reading, Massachusetts, 1984).

[3] P. Hohenberg and W. Kohn, Inhomogeneous electron gas, Phys. Rev. 136, B864 (1964).

[4] W. Kohn and L. J. Sham, Self-consistent equations including exchange and correlation effects, Phys. Rev. 140, A1133 (1965).

[5] E. H. Lieb and B. Simon, Thomas-fermi theory revisited, Phys. Rev. Lett. 31, 681 (1973).

[6] R. O. Jones, Density functional theory: Its origins, rise to prominence, and future, Rev. Mod. Phys. 87, 897 (2015).

[7] E. K. U. Gross, L. N. Oliveira, and W. Kohn, Densityfunctional theory for ensembles of fractionally occupied states. i. basic formalism, Phys. Rev. A 37, 2809 (1988).

[8] L. N. Oliveira, E. K. U. Gross, and W. Kohn, Densityfunctional theory for ensembles of fractionally occupied states. ii. application to the he atom, Phys. Rev. A 37, 2821 (1988).

[9] J. P. Perdew, R. G. Parr, M. Levy, and J. L. Balduz, Density-functional theory for fractional particle number: Derivative discontinuities of the energy, Phys. Rev. Lett. 49, 1691 (1982).

[10] E. Pastorczak, N. I. Gidopoulos, and K. Pernal, Calculation of electronic excited states of molecules using the helmholtz free-energy minimum principle, Phys. Rev. A 87, 062501 (2013).

[11] O. Franck and E. Fromager, Generalised adiabatic connection in ensemble density-functional theory for excited states: example of the $\mathrm{H}_{2}$ molecule, Mol. Phys. 112, 1684 (2014).

[12] M. Filatov, M. Huix-Rotllant, and I. Burghardt, Ensemble density functional theory method correctly describes bond dissociation, excited state electron transfer, and double excitations, J. Chem. Phys. 142, 184104 (2015).

[13] M. Filatov, Spin-restricted ensemble-referenced kohnsham method: basic principles and application to strongly correlated ground and excited states of molecules, WIREs Comput. Mol. Sci. 5, 146 (2015).

[14] M. Filatov, Ensemble DFT approach to excited states of strongly correlated molecular systems, in DensityFunctional Methods for Excited States, edited by N. Ferré, M. Filatov, and M. Huix-Rotllant (Springer International Publishing, Cham, 2016) pp. 97-124.

[15] K. Deur, L. Mazouin, and E. Fromager, Exact ensemble density functional theory for excited states in a model system: Investigating the weight dependence of the correlation energy, Phys. Rev. B 95, 035120 (2017).

[16] K. Deur and E. Fromager, Ground and excited energy levels can be extracted exactly from a single ensemble density-functional theory calculation, J. Chem. Phys. 150, 094106 (2019), https://doi.org/10.1063/1.5084312.

[17] P.-F. Loos and E. Fromager, A weight-dependent local correlation density-functional approximation for ensembles, J. Chem. Phys. 152, 214101 (2020).

[18] A. Pribram-Jones, Z.-h. Yang, J. R. Trail, K. Burke, R. J. Needs, and C. A. Ullrich, Excitations and benchmark ensemble density functional theory for two electrons, J. Chem. Phys. 140 (2014).

[19] Z.-h. Yang, J. R. Trail, A. Pribram-Jones, K. Burke, R. J. Needs, and C. A. Ullrich, Exact and approximate kohnsham potentials in ensemble density-functional theory, Phys. Rev. A 90, 042501 (2014). 
[20] Z.-h. Yang, A. Pribram-Jones, K. Burke, and C. A. Ullrich, Direct extraction of excitation energies from ensemble density-functional theory, Phys. Rev. Lett. 119, 033003 (2017).

[21] T. Gould and S. Pittalis, Hartree and exchange in ensemble density functional theory: Avoiding the nonuniqueness disaster, Phys. Rev. Lett. 119, 243001 (2017).

[22] T. Gould, L. Kronik, and S. Pittalis, Charge transfer excitations from exact and approximate ensemble kohn-sham theory, J. Chem. Phys. 148, 174101 (2018).

[23] T. Gould and S. Pittalis, Density-driven correlations in many-electron ensembles: Theory and application for excited states, Phys. Rev. Lett. 123, 016401 (2019).

[24] T. Gould and S. Pittalis, Density-driven correlations in ensemble density functional theory: Insights from simple excitations in atoms, Aust. J. Chem. 10.1071/ch19504 (2020).

[25] E. Fromager, Individual correlations in ensemble density functional theory: State- and density-driven decompositions without additional kohn-sham systems, Phys. Rev. Lett. 124, 10.1103/physrevlett.124.243001 (2020).

[26] C. Marut, B. Senjean, E. Fromager, and P.-F. Loos, Weight dependence of local exchange-correlation functionals in ensemble density-functional theory: double excitations in two-electron systems, Faraday Discuss. 10.1039/d0fd00059k (2020).

[27] T. Gould, Approximately self-consistent ensemble density functional theory: toward inclusion of all correlations, ChemRxiv 10.26434/chemrxiv.12382595.v1 (2020).

[28] E. K. U. Gross, L. N. Oliveira, and W. Kohn, Rayleighritz variational principle for ensembles of fractionally occupied states, Phys. Rev. A 37, 2805 (1988).

[29] M. Perarnau-Llobet, K. V. Hovhannisyan, M. Huber, P. Skrzypczyk, N. Brunner, and A. Acín, Extractable work from correlations, Phys. Rev. X 5, 041011 (2015).

[30] By totally symmetric we mean, as usual, ensembles which are invariant under any symmetry operations of the considered Hamiltonian [24].

[31] To avoid ensemble $v$-representability concerns we consider only "well-behaved" densities here for which $v_{s}[n]$ exists.

[32] This is a highly desirable prerequisite especially when dealing with multiplets.

[33] N. I. Gidopoulos, P. G. Papaconstantinou, and E. K. U. Gross, Spurious interactions, and their correction, in the ensemble-kohn-sham scheme for excited states, Phys.
Rev. Lett. 88, 033003 (2002).

[34] E. Pastorczak and K. Pernal, Ensemble density variational methods with self- and ghost-interaction-corrected functionals, J. Chem. Phys. 140, 18A514 (2014).

[35] Note, this assumption is usually made implicitly in KohnSham EDFT. The results from this work thus may not apply in general but certainly cover all common applications of EDFT.

[36] Supplementary material may be found at XXXXXXXXX.

[37] Gould and Kronik, Ensemble Generalized KohnSham Theory: The Good, the Bad, and the Ugly, chemrxiv.12846836.v1 10.26434/chemrxiv.12846836.v1 (2020).

[38] R. T. Sharp and G. K. Horton, A variational approach to the unipotential many-electron problem, Phys. Rev. 90, 317 (1953).

[39] J. D. Talman and W. F. Shadwick, Optimized effective atomic central potential, Phys. Rev. A 14, 36 (1976).

[40] Á. Nagy, An alternative optimized potential method for ensembles of excited states, J. Phys. B: At., Mol. Opt. Phys. 34, 2363 (2001).

[41] J. F. Dobson, K. McLennan, A. Rubio, J. Wang, T. Gould, H. M. Le, and B. P. Dinte, Prediction of dispersion forces: Is there a problem?, Aus. J. Chem. 54, $513(2001)$.

[42] J. F. Dobson and T. Gould, Calculation of dispersion energies, J. Phys.: Condens. Matter 24, 073201 (2012).

[43] H. Eshuis, J. E. Bates, and F. Furche, Electron correlation methods based on the random phase approximation, Theor. Chem. Acc. 131, 1 (2012).

[44] X. Ren, P. Rinke, C. Joas, and M. Scheffler, Randomphase approximation and its applications in computational chemistry and materials science, J. Mater. Sci. 47, 7447 (2012).

[45] J. P. Perdew, Jacob's ladder of density functional approximations for the exchange-correlation energy, in AIP Conference Proceedings (AIP, 2001).

[46] N. T. Maitra, F. Zhang, R. J. Cave, and K. Burke, Double excitations within time-dependent density functional theory linear response, J. Chem. Phys. 120, 5932 (2004).

[47] P. Elliott, S. Goldson, C. Canahui, and N. T. Maitra, Perspectives on double-excitations in TDDFT, Chem. Phys. 391, 110 (2011). 